\title{
Immunodetection of Cytoplasmatic Membrane-Bound Thrombomodulin in Formalin-Fixed Paraffin-Embedded Human Tissue Microarrays
}

\author{
Ruddock MW ${ }^{1 *}$, Reid $\mathrm{CN}^{1}$, McReynolds $\mathrm{D}^{1}$, Lamont $\mathrm{JV}^{1}$, Fitzgerald $\mathrm{SP}^{1}$ and Williamson $\mathrm{KE}^{2}$ \\ ${ }^{1}$ Randox Laboratories Ltd, Molecular Biology, 55 Diamond Road, Crumlin, County Antrim, Northern Ireland, UK \\ ${ }^{2}$ Centre for Cancer Research and Cell Biology (CCRCB), Queens University Belfast, 97 Lisburn Road, Belfast, County Antrim, Northern Ireland, UK
}

"Corresponding author: Mark W Ruddock, Randox Laboratories Ltd, Molecular Biology, 55 Diamond Road, Crumlin, County Antrim, Northern Ireland, UK, Tel: +44 (0) 289442 2413; Fax: +44 (0) 289445 2912; E-mail: mark.ruddock@randox.com

Rec Date: August 4, 2014, Acc date: October 14, 2014, Pub date: October 16, 2014

Copyright: @ 2014 . Ruddock MW, et al. This is an open-access article distributed under the terms of the Creative Commons Attribution License, which permits unrestricted use, distribution, and reproduction in any medium, provided the original author and source are credited.

\begin{abstract}
Background: Loss of thrombomodulin immunoreactivity denotes a poor prognosis for patients presenting with malignant disease.

Objectives: Immunodetection for cytoplasmatic membrane-bound thrombomodulin in formalin-fixed paraffinembedded (FFPE) tissue microarrays.

Methods: Thrombomodulin immunoreactivity was assessed in 97 FFPE tissue cores representing bladder, kidney, prostate, testis, penis, ovary, cervix, vulva, endometriosis, and myometrium, from 84 patients arrayed on three TMAs (64 (66\%) cancers, $22(23 \%)$ normal controls, and $11(11 \%)$ benign pathologies). Human bladder tissue lysate from matched normal urothelium and TCC biopsies were also examined by western blot analysis for thrombomodulin expression.
\end{abstract}

Results: Thrombomodulin immunoreactivity was strongest in the TCC cores where $\geq 60 \%$ of the tissue sections scored +3 , or greater. By contrast, SCCs, adenocarcinomas, CCCs, papillary carcinoma, sarcomas, seminomas, teratoma and all other tissue sections scored $\leq+2$. Thrombomodulin expression was also detected by western blot analysis in the human bladder tumour lysate. No signal was detected in adjacent normal control.

Conclusion: Cytoplasmatic membrane-bound thrombomodulin immunostaining is strongest in TCC sections with respect to SCC, adenocarcinoma, sarcoma, seminoma, teratoma, papillary carcinoma, CCC, and is independent of both grade and stage. Thrombomodulin immunostaining in tumour tissue sections are predominately membranous.

Keywords: Bladder; Tumour tissue microarray; Thrombomodulin; Immunostaining; Transitional cell carcinoma; Squamous cell carcinoma; Adenocarcinoma; Urothelial cancer

\section{Abbreviations:}

TMA: Tissue Microarray; TCC: Transitional Cell Carcinoma; SCC: Squamous Cell Carcinoma; FFPE: Formalin Fixed Paraffin Embedded; DAB: 3,3'-diaminobenzidine

\section{Introduction}

Thrombomodulin, a $75 \mathrm{kDa}$ glycoprotein, is a membrane protein expressed on the surface of endothelial cells where it serves as a cofactor for thrombin. Thrombomodulin reduces blood coagulation by converting thrombin to an anticoagulant from a pro-coagulant enzyme [1]. Recent studies, however, have shown that thrombomodulin also affects physiological and pathophysiological systems which include anti-proliferative and anti-metastatic effects in carcinogenesis, and a loss of thrombomodulin expression has been linked to a poor prognosis [2].

Manning et al., have described an inverse correlation with thrombomodulin expression with cancer progression and metastasis
[3]. However, in direct contrast, Ding et al., reported that thrombomodulin was up-regulated in bladder cancer patients and cancer cell lines, but not in normal cells [4]. Furthermore, these authors also claim that thrombomodulin immunostaining in bladder cancer TMAs were more pronounced in high grade and invasive cancers.

Identification of poorly differentiated tumours originating in the renal pelvis can prove challenging due to the numerous histological variants [5]. As such, thrombomodulin has been suggested as a potential biomarker that could be useful in the differential diagnosis of urothelial cancers $[5,6]$.

\section{Materials and Methods}

\section{Materials}

Human bladder cancer urinary tissue microarrays (TMAs) (TMA2205/2206/2207) and human bladder tissue lysate (normal (T10-003-N-1) and tumour (T10-003-T-1)) were purchased from Protein Biotechnologies, Ramona, CA92065. Tumour grading and staging information accompanied the TMAs and lysate provided by Protein Biotechnologies. Rabbit monoclonal anti-thrombomodulin [EPR4051] antibody (AB109189), HistoReveal (AB103720) and DAB 
Citation: Ruddock MW, Reid CN, Reynolds DM, Lamont JV, Fitzgerald SP, et al. (2014) Immunodetection of Cytoplasmatic Membrane-Bound Thrombomodulin in Formalin-Fixed Paraffin-Embedded Human Tissue Microarrays. J Mol Genet Med 8: 140. doi: $10.4172 / 1747-0862.1000140$

Page 2 of 5

substrate kit (AB64238) were obtained from Abcam, UK. Clearing reagent Histo-Clear (HS-200) was obtained from National Diagnostics, Atlanta, Georgia, US. Bovine serum albumin (BSA) (A4503), goat anti-rabbit HRP (A0545), absolute ethanol (24103), and counterstain, Harris haematoxylin (HHS32) were obtained from Sigma, Poole, UK. Hydrogen peroxide 30\% (95321) was obtained from Fluka, UK. All other reagents, unless otherwise indicated, were obtained from Sigma, Poole, UK.

\section{Study population}

We investigated thrombomodulin immunoreactivity in 97 FFPE tissue samples including bladder, kidney, prostate, penis, testis, cervix, vulva, endometrium and ovary from 84 patients. Fifty-two were males (61.9\%) (Age $54.8 \pm 17.2$, median age 56) and 32 females (38.1\%) (Age $47.5 \pm 12.6$, median age 49.5). We assessed samples from 22 patients (22/97 (22.7\%)) with histologically "normal" tissue; 11 patients (11/97 (11.3\%)) with benign pathologies consisting of $2 / 97$ (2.1\%) chronic cystitis bladder, $2 / 97$ (2.1\%) benign prostate hyperplasia (BPH), 5/97 (5.1\%) benign pathologies of the kidney, 1/97 (1\%) cervical polyps, and 1/97 (1\%) with leiomyoma myometrium. The remaining 64/97 (66\%) were transitional cell carcinoma of the bladder 35/97 (36.1\%); squamous cell carcinoma $7 / 97(7.2 \%)$ of the bladder $(\mathrm{n}=2)$, penis $(n=1)$, cervix $(n=3)$ and vulva $(n=1)$; adenocarcinoma $14 / 97(14.4 \%)$ of the bladder $(n=6)$, prostate $(n=2)$, ovary $(n=3)$ and endometriosis $(\mathrm{n}=3)$; seminoma testis $2 / 97$ (2.1\%); sarcoma $2 / 97$ (2.1\%) of the bladder $(\mathrm{n}=1)$ and myometrium $(\mathrm{n}=1)$; kidney clear cell carcinoma 2/97 (2.1\%); ovarian teratoma $1 / 97(1 \%)$ and papillary carcinoma kidney $1 / 97$ (1\%).

\section{Immunohistochemistry for cytoplasmatic membrane-bound thrombomodulin}

Thrombomodulin immunostaining was performed on $4 \mu \mathrm{m}$ FFPE sections cut from three TMAs containing cores across a range of malignant and benign tissues. To demonstrate the distribution of cytoplasmatic membrane-bound thrombomodulin, we selected TMAs which covered the urinary bladder and the renal pelvis. Tissue sections were deparaffinised in Histo-Clear and rehydrated in descending concentrations (100\% to $50 \%)$ of ethanol. The slide sections were kept in tap water until ready to perform antigen retrieval. Excess water was removed from the slides, without allowing the tissue section to dry, and one or two drops of HistoReveal were applied to the TMA. The slides were then incubated for $5 \mathrm{~min}$ at RT followed by $2 \times 5 \mathrm{~min}$ washes in TBS/Tween 20 (0.1\%), with gentle agitation. The slides were then blocked for $2 \mathrm{~h}$ at RT in $10 \%$ normal serum (Randox, UK) prepared in TBS/Tween $20(0.1 \%)$. The tissue sections were drained and wiped with tissue paper prior to incubation overnight at $4^{\circ} \mathrm{C}$ with gentle rocking with the primary antibody (rabbit monoclonal antithrombomodulin) diluted $1: 100$ in TBS/Tween 20 (0.1\%), 1\% BSA. The sections from the TMAs were then rinsed three times in TBS/ Tween $20(0.1 \%)$ for $5 \mathrm{~min}$ with gentle agitation prior to incubation in $0.3 \%$ hydrogen peroxide/TBS for $15 \mathrm{~min}$. Following two further washes using TBS/Tween $20(0.1 \%)$, the TMAs were incubated with enzyme-conjugated goat anti-rabbit HRP secondary antibody, (1:2000) prepared in TBS/Tween $20(0.1 \%)$, and incubated for $1 \mathrm{~h}$ at RT and then washed in TBS/Tween $20(0.1 \%)$. Chromogen was prepared according to manufacturer's instructions and the sections from the TMAs were incubated with 3,3'-diaminobenzidine (DAB) reagent $(0.5$ $\mathrm{ml}$ ) for $10 \mathrm{~min}$ at $\mathrm{RT}$, then rinsed in running tap water for $5 \mathrm{~min}$ and counterstained with Harris haematoxylin. Following washing, the slides were dehydrated through the alcohols (50\% to $100 \%)$ up to Histo-Clear. A microscope cover glass (Erie Scientific, Portsmouth, N.H., US) $(24 \times 40 \mathrm{~mm})$ was then applied to the TMA using Eukitt $\odot$ media as the mountant. Immunoreactivity scoring for cytoplasmatic membrane-bound thrombomodulin was undertaken by two observers (MWR and CNR).

\section{Western blot analysis for the detection of thrombomodulin expression in normal and tumour tissue lysate}

Lysates from human transitional cell carcinoma, grade 2, stage II (T2NxM0)) tissue and the corresponding histologically "normal" matched urothelium from a male, aged 64 were subjected to Western blot analysis. Protein $(10 \mu \mathrm{g})$ from the matched normal and tumour lysate $(1 \mathrm{mg} / \mathrm{ml}$, respectively) were resolved on $12.5 \%$ SDS PAGE gel (300 V, 1 hour) under reducing conditions. Proteins were then transferred to nitrocellulose (BioRad, US) $(120 \mathrm{~V}, 1.5 \mathrm{~h})$. The membrane was blocked in 3\% BSA prepared in TBS/Triton X-100 $(0.025 \%)$ at $\mathrm{RT}$ for $1 \mathrm{~h}$ with gentle rocking. Primary antithrombomodulin antibody $(1: 1000)$ prepared in TBS/Triton X-100 $(0.025 \%), 1 \%$ BSA $(10 \mathrm{ml})$ was applied to the membrane which was then incubated overnight at $4^{\circ} \mathrm{C}$ with gentle rocking after which the membrane was then washed with TBS/Triton X-100 (0.025\%) $(3 \times 15$ minutes). A secondary antibody (goat anti-rabbit HRP 1:5000) prepared in TBS/Triton X-100 $(0.025 \%), 1 \%$ BSA $(10 \mathrm{ml})$ was applied to the membrane which was then incubated for a further $1 \mathrm{~h}$ at RT with gentle rocking. The membrane was washed with TBS/Triton X-100 (0.025\%) (3×15 minutes) and protein bands were visualised using enhanced chemiluminescence (ECL) solution (Roche, France).

\section{Results}

\section{Tumour grade}

The grades of the TCC $(n=35)$ were grade I $(n=6)$, grade I - II $(n=3)$, grade II $(n=10)$, grade II - III $(n=3)$, grade III $(n=10)$, tumour in situ (Tis) $(n=1)$, and 2 ungraded tissue samples. The SCC $(n=7)$, were grade I $(n=3)$, grade II $(n=2)$, grade III $(n=1)$ and one tissue section was ungraded. For the adenocarcinoma $(n=14)$, grade I $(n=1)$, grade II $(n=3)$, grade II - III $(n=2)$, grade III $(n=3)$ and five tissues were ungraded. Eight further tissue samples were also ungraded: seminoma $(n=2)$, sarcoma $(n=2)$, CCC of the kidney $(n=2)$, teratoma $(n=1)$ and papillary carcinoma of the kidney $(n=1)$.

\section{Tumour staging}

Tumour staging for the tissue samples were as follows: TCC $(n=35)$ : T1N0M0 ( $\mathrm{n}=18)$, T2N0M0 $(\mathrm{n}=11)$, T2NXM0 $(\mathrm{n}=1)$, T2N0M1 $(\mathrm{n}=1)$, T3N2M1 (n=1), T4N2MX $(n=2)$ and TisN0M0 $(n=1)$; SCC $(n=7)$ : T1N0M0 $(n=3)$, T1N1M0 $(n=2)$ and T2N0M0 $(n=2)$; adenocarcinoma $(n=14)$ : T1N0M0 $(n=7)$ and T2N0M0 $(n=7)$; seminoma $(n=2)$ : T2N0M0 $(n=1)$ and one section was not staged; kidney clear cell carcinoma $(\mathrm{n}=2)$ T1N0M0, teratoma [1] not staged; kidney papillary carcinoma $(\mathrm{n}=1)$ T1N0M0.

\section{Immunodetection for cytoplasmatic membrane-bound thrombomodulin in human TMAs}

Thrombomodulin immunoreactivity scores are summarized in Table 1. 
Citation: Ruddock MW, Reid CN, Reynolds DM, Lamont JV, Fitzgerald SP, et al. (2014) Immunodetection of Cytoplasmatic Membrane-Bound Thrombomodulin in Formalin-Fixed Paraffin-Embedded Human Tissue Microarrays. J Mol Genet Med 8: 140. doi: $10.4172 / 1747-0862.1000140$

Page 3 of 5

\begin{tabular}{|l|l|l|l|l|l|l|}
\hline \multicolumn{2}{|l|}{ Transitional cell carcinoma } \\
\hline Cases & & 0 & $1+$ & $2+$ & $3+$ & $4+$ \\
\hline CIS & 1 & & & & & 1 \\
\hline I & 6 & 1 & & 2 & 1 & 2 \\
\hline I-II & 3 & & & 1 & & 2 \\
\hline II & 10 & & 4 & 1 & 3 & 2 \\
\hline II-III & 3 & & & & 3 & \\
\hline III & 10 & & 3 & 1 & 4 & 2 \\
\hline Ungraded & 2 & & & 1 & & 1 \\
\hline$\%$ cases & & 2.8 & 20 & 17.2 & 31.4 & 28.6 \\
\hline Saumous ceI carcin| & & & & &
\end{tabular}

Squamous cell carcinoma

\begin{tabular}{|l|l|l|l|l|l|l|}
\hline I & 3 & & 3 & & & \\
\hline I-II & 0 & & & & & \\
\hline II & 2 & & 1 & 1 & & \\
\hline II-III & 0 & & & & & \\
\hline III & 1 & & 1 & & & \\
\hline Ungraded & 1 & & 1 & & & \\
\hline \% cases & & 0 & 85.7 & 14.3 & 0 & 0 \\
\hline
\end{tabular}

Adenocarcinoma

\begin{tabular}{|l|l|l|l|l|l|l|}
\hline I & 1 & & & 1 & & \\
\hline I-II II & 0 & & & & & \\
\hline II-III & 3 & & 1 & 2 & & \\
\hline III & 2 & & 1 & & & 1 \\
\hline Ungraded & 5 & 3 & 1 & 1 & & \\
\hline \% cases & & 21.4 & 35.7 & 35.7 & 0 & 7.2 \\
\hline
\end{tabular}

\section{Normal Tissue}

\begin{tabular}{|l|l|l|l|l|l|l|}
\hline Normal & 22 & 3 & 11 & 7 & 1 & \\
\hline$\%$ cases & & 13.7 & 50 & 31.8 & 4.5 & 0 \\
\hline
\end{tabular}

Benign pathologies

\begin{tabular}{|l|l|l|l|l|l|l|}
\hline Benign & 11 & 2 & 6 & 3 & & \\
\hline$\%$ cases & & 18.2 & 54.5 & 27.3 & 0 & 0 \\
\hline
\end{tabular}

Seminomas $(n=2) 1+$, sarcomas $(n=2) 0,3+$, clear cell carcinomas $(n=2) 1+$, teratoma $(n=1) 1+$, and papillary carcinoma $(n=1) 1+$.

Table 1: Immunostaining for cytoplasmatic membrane-bound thrombomodulin in human TMAs.

Thrombomodulin immunostaining on all tissue sections was undertaken by DMR. Two independent observers (MWR and CNR) scored the tissue sections for thrombomodulin immunostaining using

the EORTC-GCCG scoring system [7]. Briefly, tissue sections that were negative (score 0 ), weakly positive sections (score 1), positive sections (score 2 ), strongly positive sections (score 3 ) and very strongly positive sections (score 4). Representative micrographs for thrombomodulin immunostained TCC, SCC and adenocarcinoma, and their complementary H\&E stained sections are shown in Figure 1.

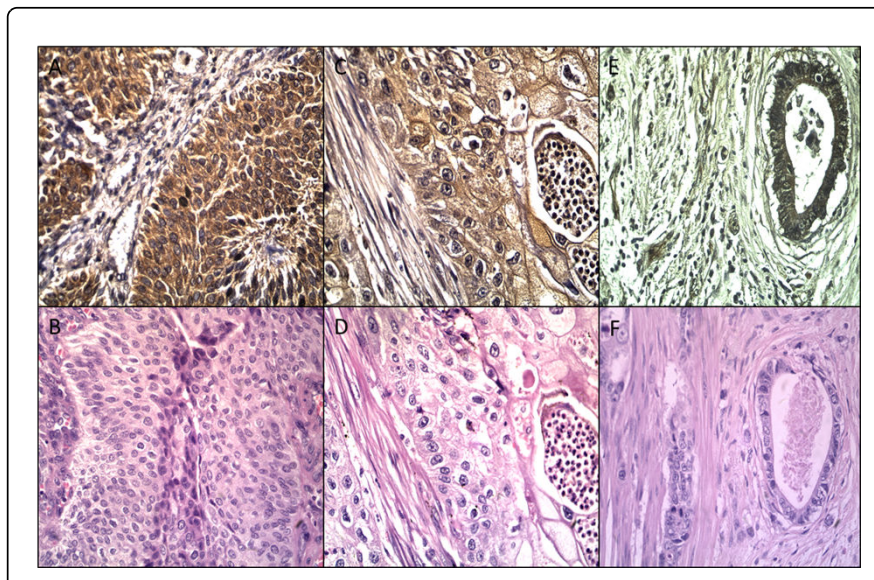

Figure 1: Immunostaining for cytoplasmatic membrane-bound thrombomodulin and an H\&E stain in human bladder tissue (i) TCC (grade 2, T3N0M0) (panel A and B,respectively), (ii) SCC (grade 2, TNOM0) (panel C and D, respectively), and (iii) adenocarcinoma (grade 2, T3N0M0) (panel E and F, respectively) TMAs. Transitional cell and SCC of the bladder (panel A and C) demonstrate strong immunoreactivity for cytoplasmatic membrane-bound thromobomodulin (scoring +3 and +2 , respectively). However, adenocarcinoma of the bladder shows weak immunoreactivity for thrombomodulin (scoring +1 ). Micrograph images were captured by an Optika B-800 series microscope using an Optika PRO3 Vision PRO digital camera (magnification $\mathrm{x} 400$ ).

Transitional cell carcinomas 34/35 (97\%), SCC $7 / 7$ (100\%), adenocarcinoma $11 / 14(78.6 \%)$, seminomas $2 / 2(100 \%)$, sarcomas $1 / 2$ (50\%), CCCs $2 / 2(100 \%)$, teratoma $1 / 1(100 \%)$, papillary carcinoma $1 / 1$ (100\%), normal urothelium 19/22 (86.4\%) and benign pathologies 9/11 (81.8\%) all displayed prominent thrombomodulin immunoreactivity. Tissue sections from TCCs displayed the strongest thrombomodulin immunoreactivity with $21 / 35(60 \%)$ of the cores scoring $>3+, 11 / 35(31.4 \%) 3+$ and $10 / 35$ (28.6\%) scoring 4+. By contrast, thrombomodulin immunoreactivity for SCC cores were all $<2+$ with $1 / 7 \quad(14.3 \%) \quad 2+$ and $6 / 7$ (85.7\%) scoring $1+$. Immunoreactivity within the cores representing adenocarcinoma displayed similar thrombomodulin immunoreactivity to that of SCCs with $5 / 14(35.7 \%) 2+, 5 / 14(35.7 \%) 1+, 3 / 14(21.4 \%) 0$ and $1 / 14(7.2 \%)$ scoring $4+$. Benign pathologies also scored $<2+$ for thrombomodulin immunoreactivity with $2 / 11$ (18.2\%) scoring $0,6 / 11(54.5 \%) 1+$ and $3 / 11(27.3 \%) 2+$. Normal tissue sections also immunostained positive for thrombomodulin in a similar fashion to that observed for the benign pathologies with $3 / 22(13.7 \%)$ scoring $0,11 / 22(50 \%) 1+, 7 / 22$ $(31.8 \%) 2+$ and $1 / 22(4.5 \%)$ scoring $3+$. In agreement with previous data [5], we did not observe any correlation between thrombomodulin immunoreactivity and the histological grade or stage of the tumour sections. 
Citation: Ruddock MW, Reid CN, Reynolds DM, Lamont JV, Fitzgerald SP, et al. (2014) Immunodetection of Cytoplasmatic Membrane-Bound Thrombomodulin in Formalin-Fixed Paraffin-Embedded Human Tissue Microarrays. J Mol Genet Med 8: 140. doi: 10.4172/1747-0862.1000140

Page 4 of 5

\section{Western blot analysis}

Normal (T10-003-N-1) and tumour (T10-003-T-1) tissue lysate from human bladder was subjected to western blot analysis, as described in the materials and methods section. A positive signal for thrombomodulin (approximately $100 \mathrm{kDa}$ ) was observed on the blot for the tumour lysate sample (Figure 2).

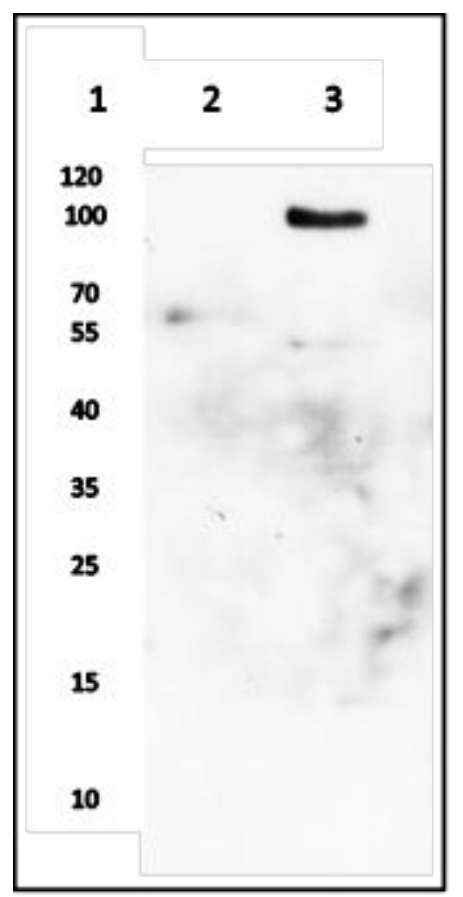

Figure 2: Immunoblot for the detection of thrombomodulin in human bladder clarified tissue lysate (normal tissue (lane 2) and tumour tissue (lane 3)) from a 64 year old male with the following pathology: TCC, grade 2, stage II, T2NxM0. Protein $(10 \mu \mathrm{g})$ from normal and bladder tumour tissue lysate $(1 \mathrm{mg} / \mathrm{ml}$, respectively) was electrophoresed on a $12.5 \%$ SDS PAGE under reducing conditions, and then transferred to PVDF membrane. The membrane was blocked with $3 \%$ BSA in TBS-T and then probed with a rabbit monoclonal anti-thrombomodulin antibody (1:1000), as described in the materials and methods section. A single band at approximately $100 \mathrm{kDa}$ was observed in lane 3, tumour tissue lysate. However, no signal was observed for thrombomodulin in the normal bladder lysate, lane 2. Molecular mass markers $(\mathrm{kDa})$ are indicated in lane 1.

No signal was observed for the normal bladder control lysate. The predicted molecular weight for thrombomodulin is approximately 75 $\mathrm{kDa}$. However, as the thrombomodulin is heavily glycosylated, the protein runs on the gel at $<100 \mathrm{kDa}$.

\section{Discussion}

Thrombomodulin is a surface glycoprotein that has been shown to be an important biomarker in patients presenting with malignant disease $[5,6]$. Decreased thrombomodulin expression is associated with cell proliferation and an increase in malignancy. Moreover, the loss of thrombomodulin expression is associated with a poor prognosis [2].
Thrombomodulin exists in two forms, a high molecular weight form which is bound to cytoplasmic membranes and a lower molecular weight form found in both plasma and urine [8]. Elevated plasma levels of thrombomodulin have been correlated with the number of years of tobacco smoking, a known risk factor for bladder cancer, which results in vascular endothelial dysfunction and shedding [9-11]. Endothelial dysfuction and shedding are common pathologies reported for patients presenting with hypertension or diabetes [12].

An increase in thrombomodulin expression in the transitional epithelium has also been reported with a worsening pathological status of cystitis [13]. Interestingly, thrombomodulin levels have been shown to differ significantly between blood groups (AB0) [7].

In this study, thrombomodulin immunostaining was observed in 88/97 (90.7\%) of the FFPE tissue sections examined. Thrombomodulin immunostaining was greater in the TCC sections with $60 \%$ of the sections scoring $\geq 3+$. However, tumour grading and staging in the TCC sections did not correlate with the intensity of thrombomodulin immunostaining, as previously reported [5]. Weak thrombomodulin immunostaining was observed in both SCCs $(6 / 7$ (85.7\%) scoring $1+)$ and adenocarcinoma $(10 / 14(71.4 \%)$ scoring $\leq 2+)$ tissue sections, and again thrombomodulin immunostaining was independent of either tumour grade or stage. Four tissue sections, 3/14 (21.4\%) adenocarcinoma and $1 / 35 \quad(2.8 \%)$ TCC were negative for thrombomodulin immunostaining. Normal and benign pathologies also demonstrated weak thrombomodulin immunoreactivity with most of the tissue sections scoring either $1+$ or $2+$. Three normal $3 / 22$ (13.7\%) and $2 / 11$ (18.2\%) benign pathologies were negative for thrombomodulin immunoreactivity.

Our data is in agreement with previous studies that suggest that thrombomodulin immunostaining may have clinical utility in differentiating between tumours arising in the renal pelvis $[5,6]$. However, in contrast to Ding et al., we did not observe an increase in thrombomodulin immunostaining with respect to either tumour grade or staging. Moreover, if the loss of thrombomodulin expression correlates with an increase in malignancy and poor prognosis, then it seems counterintuitive that thrombomodulin expression would be augmented in higher grade and higher stage tumours as suggested [4]. A recent paper by $\mathrm{Wu}$ et al. clearly demonstrates that thrombomodulin expression in bladder cancer cell lines is inversely correlated with invasive ability.

\section{Acknowledgements}

The authors acknowledge financial support from Invest Northern Ireland (INI) RD0412515.

\section{References}

1. Castellino FJ, Ploplis VA (2009) The protein C pathway and pathologic processes. J Thromb Haemost 7 Suppl 1: 140-145.

2. Wu CT, Chang YH, Lin P, Chen WC, Chen MF (2014) Thrombomodulin expression regulates tumorigenesis in bladder cancer. BMC Cancer 14: 375.

3. Manning T, Smoller BR, Horn TD, E Darouti M, Marzouk S, et al. (2004) Evaluation of anti-thrombomodulin antibody as a tumor marker for vascular neoplasms. J Cutan Pathol 31: 652-656.

4. Ding AA, Su WP, Yang WH (2011) The role of thrombomodulin in bladder cancer. Cancer Research 71: S1.

5. Ordóñez NG (1997) Value of thrombomodulin immunostaining in the diagnosis of transitional cell carcinoma: a comparative study with carcinoembryonic antigen. Histopathology 31: 517-524. 
Citation: Ruddock MW, Reid CN, Reynolds DM, Lamont JV, Fitzgerald SP, et al. (2014) Immunodetection of Cytoplasmatic Membrane-Bound Thrombomodulin in Formalin-Fixed Paraffin-Embedded Human Tissue Microarrays. J Mol Genet Med 8: 140. doi: 10.4172/1747-0862.1000140

Page 5 of 5

6. Ordóñez NG (1998) Thrombomodulin expression in transitional cell carcinoma. Am J Clin Pathol 110: 385-390.

7. Van Diest PJ, Van Dam P, Henzen-Logmans SC, Berns E, van der Burg $\mathrm{ME}$, et al. A scoring system for immunohistochemical staining: consensus report of the task force for basic research of the EORTCGCCG. European Organization for Research and Treatment of CancerGynaecological Cancer Cooperative Group. J Clin Pathol 50: 801-804.

8. Califano F, Giovanniello T, Pantone P, Campana E, Parlapiano C, et al (2000) Clinical importance of thrombomodulin serum levels. Eur Rev Med Pharmacol Sci 4: 59-66.

9. Wei Y, Zhang X, Xu L, Yi S, Li Y, et al. (2012) The effect of cigarette smoke extract on thrombomodulin-thrombin binding: an atomic force microscopy study. Sci China Life Sci 55: 891-897.

10. Dohi Y, Ohashi M, Sugiyama M, Takase H, Sato K, et al. (2003) Circulating thrombomodulin levels are related to latent progression of atherosclerosis in hypertensive patients. Hypertens Res 26: 479-483.
11. Jansson JH, Boman K, Brännström M, Nilsson TK (1996) Increased levels of plasma thrombomodulin are associated with vascular and allcause mortality in patients on long-term anticoagulant treatment. Eur Heart J 17: 1503-1505.

12. Münzel T, Sinning C, Post F, Warnholtz A, Schulz E (2008) Pathophysiology, diagnosis and prognostic implications of endothelial dysfunction. Ann Med 40: 180-196.

13. Obama H, Obama K, Takemoto M, Soejima Y, Shirahama T, et al. (1999) Expression of Thrombomodulin in the epithelium of the urinary bladder: a possible source of urinary thrombomodulin. Anticancer Res 19: 1143-1147. 